\title{
Organizaciones Solidarias Creadas por población vulnerable: un estudio de su planificación y gestión interna
}

\author{
Madelín Sánchez-Otero ${ }^{1}$, Migdalia Caridad-Faria ${ }^{2}$, Mildred Garizabal-Donado ${ }^{3}$ y Hugo Hernández-Palma ${ }^{4}$ \\ (1) Dpto. de Gestión Organizacional, Universidad de la Costa, CUC, Barranquilla-Colombia \\ (2) Fac. de Ciencias Administrativas, Institución Universitaria Tecnológico de Antioquia, Medellín-Colombia \\ (3) Prog. de Administración de Empresas, Corporación Universitaria Rafael Núñez, Cartagena- Colombia \\ (4) Fac. Ciencia Económicas, Universidad del Atlántico, Barranquilla - Colombia \\ (e-mail:msanchez6@cuc.edu.co; migdalia.caridad@tdea.edu.co; mildred.garizabal@virtual.edu.co; \\ hugohernandezp@mail.uniatlantico.edu.co)
}

Recibido Abr. 18, 2018; Aceptado Jun. 19, 2018; Versión final Ago. 14, 2018, Publicado Abr. 2019

\begin{abstract}
Resumen
El objetivo del trabajo presentado fue analizar la planificación y gestión interna de organizaciones de economía solidaria establecidas por población vulnerable. Se emplearon técnicas mixtas de recolección de datos, dirigidas a asociados y directivos para evaluar procesos internos que influyen en la productividad y oportunidad de inclusión social de las empresas estudiadas. Los principales hallazgos indican que la planificación es incipiente y los resultados de la gestión interna son limitados por diversos factores socioeconómicos, siendo significativo el bajo nivel de formación. Se concluye que a pesar de que las organizaciones cuentan con el apoyo de profesionales externos en el diseño y puesta en marcha de la planeación, los directivos adolecen de competencias necesarias para gestionar la ejecución de estos procesos y de recursos económicos que les permitirían lograr un mejor desempeño.
\end{abstract}

Palabras clave: gestión; organizaciones solidarias; planeación; población vulnerable

\section{Solidarity economy organizations in vulnerable populations: a reflection on challenges and planning challenges}

\begin{abstract}
The objective of this paper is to analyze the internal planning and management of solidarity economy organizations established by vulnerable populations. Mixed data collection techniques were used, aimed at associates and managers to evaluate internal processes that influence the productivity and opportunity for social inclusion of the companies studied. The main findings indicate that planning is incipient and the results of internal management are limited by various socio-economic factors, being low level training one of the most significant ones. It is concluded that although the organizations have the support of external professionals in the design and implementation of planning, managers lack the necessary skills to manage the implementation of these processes and economic resources that would allow them to achieve better performance.
\end{abstract}




\section{INTRODUCCIÓN}

Los grandes desafíos que enfrentan las economías a nivel mundial producto de los cambios permanentes en el sistema social, cultural, económico y político, han ampliado la brecha socioeconómica entre los miembros de una sociedad cada vez más competitiva. Por tanto, el ideal propuesto por los Objetivos del Desarrollo Sostenible (ODS) implica para los gobiernos un gran reto en la búsqueda de soluciones para reducir esas diferencias marcadas de origen socioeconómico que han impulsado la promulgación de políticas sociales dirigidas a los más pobres; en tal sentido, Colombia en su política pública social, se orienta a promover programas y proyectos que beneficien a personas que han sido víctimas de desplazamiento y desaparición por la situación de violencia y conflicto que se ha dado en el país, recibiendo la condición de personas vulnerables.

Las razones de su vulnerabilidad se generan porque han vivido alguna situación de conflicto armado interno, disturbios y tensiones interiores, violencia generalizada, violaciones masivas de derechos humanos, infracciones al derecho internacional humanitario $u$ otras circunstancias emanadas de las situaciones anteriores, que puedan alterar o que alteren drásticamente el orden público (Ley 387 de 1997). Esta situación de desplazamiento y las condiciones de vulnerabilidad de diversos grupos en Colombia, ha generado un sinnúmero de leyes, decretos, regulaciones y jurisprudencias, estableciendo así el marco jurídico que permite abordar la problemática, entre los cuales se destacan: la jurisprudencia 1635 y T-025 del 2004 emitida Corte Constitucional; el Decreto 250 de febrero del 2005; Ley 1448 y Decretos 4633, 4634, 4635,4800 y 4829 de 2011; la Ley 1532 de junio y el Decreto 0790 de 2012; los Conpes 3712 de 2011, 3726 de 2012 y 3784 de 2013; y la Resolución 01126 de 2015.

Por lo expuesto, surge la necesidad de que el Estado apoye al sector productivo a través de la creación de valor compartido y aliados con la academia para generar elementos teóricos y prácticos que contribuyan al crecimiento de las organizaciones solidarias y de sus miembros, mediante la creación, financiamiento y consolidación de dichas organizaciones para que sean sostenibles en el tiempo. Se responde a esta exigencia con proyectos financiados por el Estado y ejecutados por organizaciones de la sociedad civil. Al respecto Heras (2014), afirma que en la literatura se muestra solo el lado positivo de estas organizaciones y no la realidad sobre la democracia y participación en su gestión y tampoco el verdadero impacto que tienen en la sociedad. En oportunidades los asociados perciben a la organización como una entidad prestadora de servicios y no, así como organización que mediante la asociatividad se consolida en pro de todos los asociados y su núcleo familiar (Hernández y Ricardo, 2016). Como resultado de la caracterización de esta situación surge el interés en analizar a las organizaciones de economía solidaria creadas o gestadas por poblaciones vulnerables respecto a su planificación y gestión en los departamentos Atlántico, Bolívar y Magdalena respectivamente.

El estudio fue descriptivo - explicativo y la población estuvo representada por 40 organizaciones creadas en el año 2013 e impulsadas por la Unidad Administrativa Especial de Organizaciones Solidarias en los departamentos citados, las cuales en su mayoría realizan actividades económicas relacionadas con el campo y la pesca. Adicionalmente, es poca la literatura que se ha desarrollado específicamente con el enfoque en mención, autores como Lang y Ramírez (2017); Papke y Boyer (2017); Sommerfeldt y Yang (2017); Svensson (2017); Suárez et al. (2016); Vishnevskiy et al. (2016), si bien, realizan trabajos sobre planificación y gestión, su enfoque se orienta a diferentes áreas bien sea de innovación, tecnología o deportes, o con organizaciones en condiciones socio-económicas distintas al enfoque planteado en este artículo.

Por otro lado, existen trabajos donde se investiga la gestión de las organizaciones de economía solidaria, encontrando que los modelos de negocios cooperativos dependen de los roles de los miembros y su eficiencia (Chareonwongsak, 2017), también, se encontraron ejemplos de éxito en asociaciones de empresa comercial nacional con una cooperativa en Ethiopia, ello cuando se tiene una combinación de varios factores como asistencia técnica, apoyo de la empresa exportadora y una ONG. Al igual que en cooperativas agrícolas lideradas solo por mujeres en Uganda que tuvieron un impacto positivo en el bienestar económico, gestión del conocimiento y adopción de prácticas agronómicas, a pesar de lo cual las mujeres siguen teniendo desventajas en lo que respecta a divisiones laborales internas y en el hogar (Royer et al., 2017; Lecoutere, 2017).

Esta dinámica lo que busca es la participación de la sociedad en pro de la construcción de la paz duradera (Mariño y Valencia, 2015), haciendo frente a la situación de vulnerabilidad de este grupo de personas, sin embargo, Cárdenas et al. (2014), indican que este proceso se presenta desarticulado entre los actores que intervienen, existe falta de información de toda la oferta a esta población, y por parte de ésta última desconfianza en las instituciones. No obstante, esta situación representa una oportunidad para explotar la posibilidad de articulación de la política pública, las instituciones, las estrategias adecuadas para lograr el desarrollo necesario a través de la gestión social y buena gobernanza, y la vinculación estratégica y el protagonismo de la sociedad civil o de los actores involucrados (Giordano y Pereira, 2011; Garizabal et al., 2017). 
Las organizaciones son sistemas sociales compuestos por individuos que se interrelacionan entre sí y con el contexto, de tal manera que lo realizado por alguno de los actores afecta, de un modo u otro, a los demás integrantes del conjunto, donde las personas intercambian y comparten conocimientos y se construye nuevo conocimiento, tácito, explícito o virtual. Significa entonces, que en el marco de la concepción de una organización hay elementos claves como: es un sistema social, están inmersos los individuos; conocimiento que poseen los individuos que conforman la organización; se establecen objetivos a alcanzar, se dan relaciones internas y externas (Garzón, 2015); potenciando el talento de los directivos y miembros en este tipo de organización se podría dinamizar la mejora del desempeño de las mismas, por los conocimientos y el trabajo colaborativo (Álvarez y Bernal, 2017).

Sin embargo, estas organizaciones aún tienen un largo camino por recorrer, presentan diversas dificultades, entre las cuales se destacan: barreras estructurales para el éxito cooperativo, las exigencias de la gestión interna, carecen de liderazgo, de participación, redes de relaciones entre organizaciones, accionar local y debilidad en la gestión de los diversos grupos de interés (Cheney et al, 2014; Yildiz et al., 2015). Estas dificultades, también se evidencian en los estudios Alves et al., (2017) en Brasil, el cual, muestra debilidades en las habilidades para gestionar y seguir los principios cooperativos y el de Bretos y Errasti (2018) sobre la cooperativa Mondragón, estudio que evidencia relaciones de poder e interés particular de los miembros de la sede central y por otro lado, disociación del discurso público como organización de participación y democrática y de la realidad de sus prácticas internas.

Desde este contexto, la planificación debe ser un instrumento de gestión que permita la toma de decisiones comprometida con resultados, que facilite el adecuarse a los cambios y a las demandas del entorno y a la vez contribuya en la identificación de objetivos de carácter prioritario y los cursos de acción (estrategias), logrando con ello mayor eficiencia, eficacia y calidad en los bienes y servicios que se proveen (Iglesias, 2010). Igualmente, la planificación se entiende como la dinámica o proceso que realizan los directivos de una organización con la finalidad de identificar la situación actual y tomar decisiones para proyectar hacia al futuro (Serna, 2008). En este orden de ideas, la gestión tiene un enfoque de integración, en tanto, suma las fuerzas de los miembros que hacen parte de una organización (Hernández et al., 2017) logrando la sinergia de aspectos tangibles e intangibles para el logro de los objetivos de la organización. Por lo tanto, la gestión tiene como finalidad la coordinación de la totalidad de la organización y la articula con el entorno (Dávila, 2012).

La gestión se asocia a individuos o grupos de personas, quienes dirigen y coordinan recursos, talentos, situaciones adversas y oportunidades, permitiendo de esta manera la sostenibilidad de la organización. Enfatizando, la sostenibilidad plantea desafíos políticos, regulatorios, organizativos y financieros a todas las organizaciones, también, las gestadas por las sociedad civil (Hailey y Salway, 2016) y en países de ingresos bajos, la sostenibilidad, presenta mayor desafío para estas organizaciones (Arhin et al., 2018). Desde esta perspectiva, se requiere de personas con las capacidades necesarias para transformas ideas en realidades a través de acciones concretas, el desarrollo de capacidades de la organización se asocia a la gestión confiable que deben hacer sus miembros.

De los fundamentos teóricos expuestos, se evidencian elementos coincidentes de la variable gestión, entre los cuales están: lograr integrar diversos elementos, establecer metodologías de trabajo y las acciones necesarias para la consecución de los objetivos que se ha trazado la organización y de esta forma llevar a un nivel superior de crecimiento, posicionamiento en su entorno y desarrollar ventajas competitivas. Se debe considerar que la gestión se asocia con la acción, es decir, esta hace posible en una organización que los objetivos se traduzcan en resultados, la forma en que operan los directivos de algunas empresas entre sí, puede ser independiente e interdependiente (Hambrick, 2015), afectando así los resultados de gestión. La permanencia y heterogeneidad del equipo de alta gerencia hacen parte de los elementos que moderan la relación entre la estrategia y el desempeño organizacional (Chin, 2016). Para que las organizaciones puedan avanzar en función de los requerimientos del entorno, la integración con el mismo debe estar presente en cada una de las etapas de direccionamiento estratégico: el cambio, la mejora continua, la innovación tecnológica y la gestión administrativa (Hernández y del Rio, 2017).

Por otro lado, Cardozo (2011) resalta que el diseño organizacional, la filosofía de gestión, el diseño de cargos, las prácticas operativas, las estrategias de control de calidad deben hacer parte de la propuesta para hacer más competitivas las empresas de ordenamiento horizontal. En el contexto de la gestión de empresas sociales se hace necesario que se logre sinergia de las dimensiones económicas y sociales, lo cual, implica "llevar implícitos principios éticos, información accesible, oportuna, fiable y recurrente" (Caridad et al., 2014) por la naturaleza de estas organizaciones y generalmente quienes gestionan tienen doble papel en la organización, el de empleado y asociado. 


\section{METODOLOGÍA}

Para el presente estudio se optó por una investigación descriptiva - explicativa, de carácter cualitativo, enmarcada teóricamente en la ciencia administrativa, la información se obtuvo mediante grupos focales y encuestas dirigidas a asociados, entrevistas a profundidad a directivos y observación a los participantes. Se asume la postura que entre el método cuantitativo y cualitativo de investigación no hay una separación tan amplia, por el contrario, se articulan, facilitan una visión holística e integral en el análisis racional crítico de la situación problema abordada, generando resultados más confiables. La población estuvo representada en 40 organizaciones creadas en el año 2013 e impulsadas por la Unidad Administrativa Especial de Organizaciones Solidarias que en su mayoría realizan actividades económicas relacionadas con el campo y la pesca, en un rango de 10 a 100 socios por empresa.

\section{RESULTADOS}

En este aparte se describen las principales evidencias o hallazgos resultado del estudio de campo, inicialmente sobre los miembros o asociados y luego sobre las organizaciones. Desde este propósito, se identifica en la figura 1, aspectos relacionados con los miembros de la organización así: la mayoría son mujeres (65\%), la condición de vulnerabilidad de mayor incidencia es el desplazamiento (70\%) y el (2\%) de los participantes tiene formación de pregrado y técnica (7\%), es decir, el grado de formación de los participantes es bajo, lo cual, podría estar asociado su origen rural y a las condiciones de vulnerabilidad que viven.

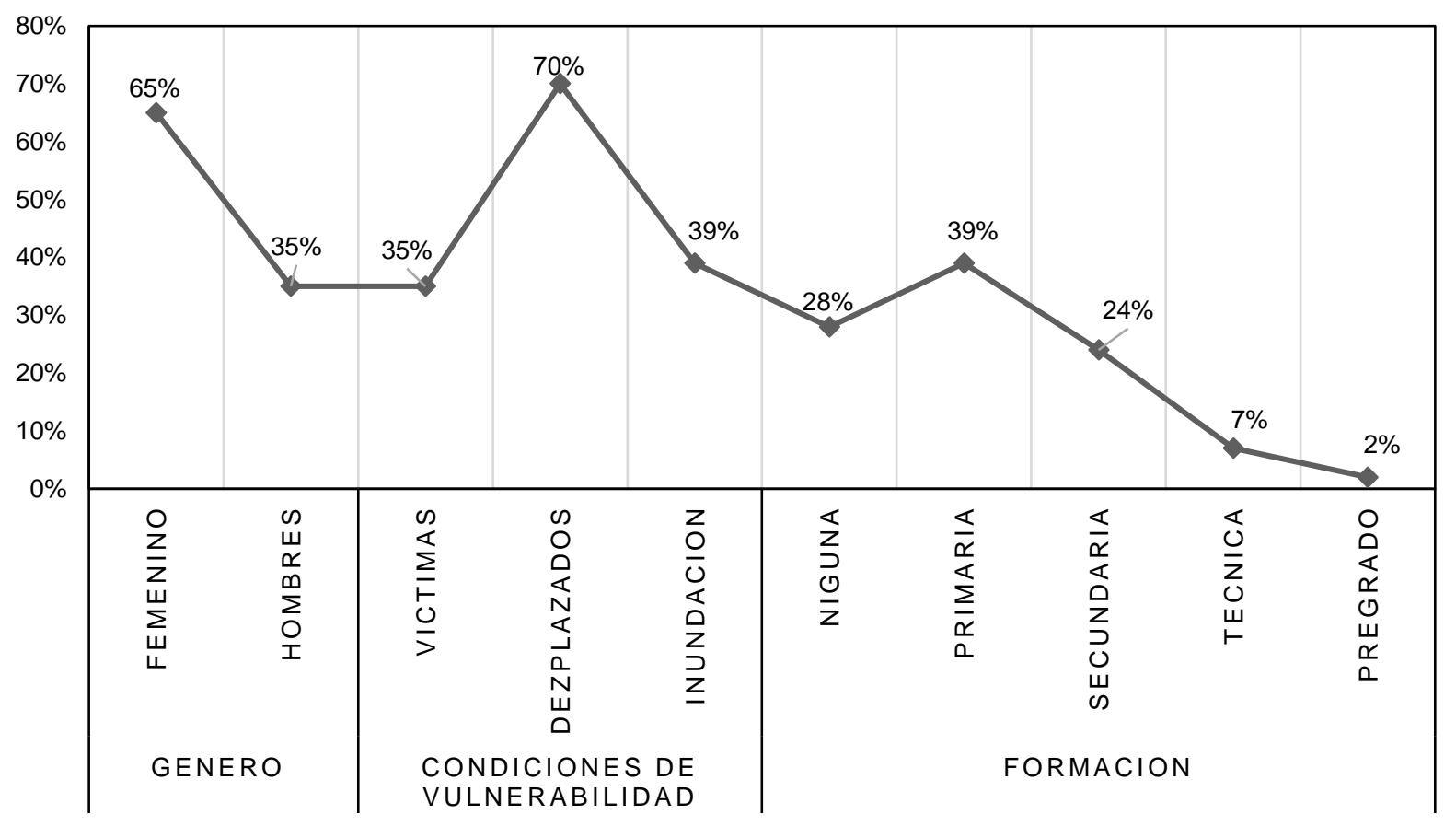

Fig.1: Generalidades de los miembros de las organizaciones en estudio

Siguiendo con el análisis de los datos, se observa en la tabla 1 que, la motivación de la mayoría de los asociados para ingresar a la cooperativa fue por invitación de alguien y, en segundo lugar, porque vendería mucho más. Asimismo, entre las dificultades que señalaron con mayor importancia fueron: falta de recursos de toda clase, no se ven beneficios reales de asociarse y organizaciones externas que solo capacitan y $\sin$ resultados reales, lo que obedece a la intervención de varias universidades y organizaciones sin ánimo de lucro trabajando con las organizaciones en los mismos temas.

Esta línea de resultados, coincide con el estudio de Iglesias (2017), el cual evidencia la desarticulación de los diferentes actores en este tipo de acciones. Con respecto si la planeación mejora la situación de las organizaciones, en su mayoría consideran que si, sin embargo, otros asociados (40\%), manifestaron que se queda en el papel, adolecen de recursos y los directivos carecen de las habilidades para llevarlo a la realidad. Por otro lado, solo el (20\%) aportaría al proceso porque el (80\%) restante están desmotivados y el tiempo que disponen es nulo, porque "solo capacitaciones y no hay recursos". A partir de estos resultados, se evidencia, la importancia del rol y eficiencia de los miembros en la funcionalidad de los modelos cooperativos 
planteado por Chareonwongsak (2017) que se centra en el proceso de planeación. Al observar los hallazgos de la tabla 2, relacionados con la percepción y expectativas de los directivos respecto al proceso de planeación y gestión se detallan los siguientes aspectos: el directivo es seleccionado por el grupo de asociados, tal vez porque lo identifican como líder; asimismo, se infiere que la organización funciona de forma intermitente, ya sea por el nivel de compromiso (no pago de aportes, individualismo, desmotivación) o porque no hay funciones definidas o los órganos de dirección establecidos en este tipo de organización no operan formalmente. También, se evidencia que los directivos son conscientes de las debilidades que tienen en habilidades y recursos para el proceso de planeación y gestión, aspectos que coinciden con los resultados que se obtuvo del grupo de asociados. La tendencia de los resultados de esta investigación, apoyan los aspectos planteados por Cheney et al. (2014), para fortalecer las organizaciones y el sector solidario.

La tendencia de los resultados descritos, también, se relaciona con la información contenida en la figura 2, concerniente a las organizaciones estudiadas desde sus resultados. Al respecto, los directivos (50\%) evidencian como uno de los principales resultados: problemáticas con el Estado, relacionados con el hecho de no declarar o renovar cámara de comercio, porque, aunque no tengan movimiento, deben cumplir con estos aspectos, situación que genera multas. El otro 50\%, considera que no se alcanzó ningún resultado y solo se reúnen para conversar de lo que han logrado. Esta línea de resultados, reafirma, lo encontrado en las investigaciones de Cheney et al. (2014); Yildiz et al. (2015); Alves et al., (2017) y Bretos y Errasti (2018), respecto a desafíos estructurales, de gestión interna y externa, de liderazgo y vinculación efectiva de grupos interés que tienen las organizaciones cooperativas. Desde estos argumentos, los procesos de planeación requieren de habilidades directivas para lograr establecer situaciones actuales y tomar decisiones proyectadas, como lo afirma Serna (2008). En las tablas 1 y 2 se aprecian los datos previamente reseñados. Seguidamente se pueden observar en la figura 2 los resultados obtenidos en las organizaciones solidarias:

Tabla 1: Situación y expectativas de los miembros o asociados respecto al proceso de planeación

\begin{tabular}{|c|c|c|}
\hline Aspectos & Respuesta & $\%$ \\
\hline \multirow{3}{*}{$\begin{array}{l}\text { Motivo de vinculación } \\
\text { a la organización }\end{array}$} & Alguien me hablo del proyecto & 100 \\
\hline & Vendería mas & 93 \\
\hline & Pensé que tendría un sueldo & 66 \\
\hline \multirow{7}{*}{$\begin{array}{l}\text { Dificultades que tiene } \\
\text { la organización }\end{array}$} & Nos falta recursos de toda clase & 100 \\
\hline & No hemos visto cambios y mejora en nuestra situación & 99 \\
\hline & No funcionamos como una empresa, nos vemos cuando hay reunión & 90 \\
\hline & No estamos preparados para gestionar la organización & 88 \\
\hline & No es buena la calidad del producto o servicio & 95 \\
\hline & No hemos vistos los beneficios reales de asociarnos & 100 \\
\hline & $\begin{array}{l}\text { Aquí vienen diferentes organizaciones a llenarnos de capacitación, } \\
\text { pero nada más }\end{array}$ & 100 \\
\hline \multirow{2}{*}{$\begin{array}{l}\text { Expectativas sobre la } \\
\text { organización }\end{array}$} & Que sea grande & 80 \\
\hline & Que venda a todas partes & 20 \\
\hline \multirow[t]{2}{*}{$\begin{array}{l}\text { Proceso de } \\
\text { planeación mejora la } \\
\text { situación }\end{array}$} & $\begin{array}{l}\text { No. Según ellos "se queda en un papel, no tenemos recursos para } \\
\text { llevarlo a cabo, los que dirigen la organización no están preparados, } \\
\text { la organización funciona cuando hay algún problema, no todos } \\
\text { aportamos de las mismas formas" }\end{array}$ & 40 \\
\hline & Si & 60 \\
\hline \multirow[t]{2}{*}{$\begin{array}{l}\text { Su aporte como } \\
\text { asociado al proceso }\end{array}$} & $\begin{array}{l}\text { Indicaron que no saben, porque "tenemos que producir para poder } \\
\text { cumplir con lo básico de la casa. Y a veces no queda tiempo para } \\
\text { venir a estas reuniones. Además, ya hemos perdido tiempo en } \\
\text { capacitación, todos estos proyectos solo dan capacitación y más } \\
\text { capacitación y uno no ve que mejore la vida de uno y eso desmotiva. } \\
\text { Tampoco tenemos los recursos necesarios para que eso se logre, el } \\
\text { estado no da dinero para maquinaria ni nada de eso" }\end{array}$ & 80 \\
\hline & $\begin{array}{l}\text { Manifestaron que podría haber un cambio o mejorar y su aporte seria } \\
\text { en asistir a las actividades programadas }\end{array}$ & 20 \\
\hline
\end{tabular}


Tabla 2: Percepción y expectativas de los directivos respecto al proceso de planeación

\begin{tabular}{|c|c|c|}
\hline Aspectos & Respuesta & $\%$ \\
\hline \multirow{3}{*}{$\begin{array}{l}\text { Elección como } \\
\text { directivo }\end{array}$} & El grupo me escogió, no me postule & 69 \\
\hline & Me ofrecí para dirigir & 20 \\
\hline & $\begin{array}{l}\text { he liderado procesos en la comunidad y eso me } \\
\text { ayudo }\end{array}$ & 11 \\
\hline \multirow{6}{*}{$\begin{array}{l}\text { Dificultades que } \\
\text { tiene la } \\
\text { organización }\end{array}$} & $\begin{array}{l}\text { No todos los asociados tienen el mismo nivel de } \\
\text { compromiso }\end{array}$ & 78 \\
\hline & $\begin{array}{l}\text { Muchos asociados se han desmotivado porque } \\
\text { quieren ver resultados ya }\end{array}$ & 90 \\
\hline & Cada quien está pensando en su bienestar & 83 \\
\hline & $\begin{array}{l}\text { El pago de aportes no es normalizado y con eso es } \\
\text { que la organización se financia }\end{array}$ & 91 \\
\hline & $\begin{array}{l}\text { No tenemos garantías para que nos den un } \\
\text { préstamo }\end{array}$ & 100 \\
\hline & $\begin{array}{l}\text { No funcionamos como una empresa, solo nos } \\
\text { reunimos cuando hay algo que discutir }\end{array}$ & 95 \\
\hline \multirow{2}{*}{$\begin{array}{l}\text { Expectativas } \\
\text { sobre la } \\
\text { organización }\end{array}$} & Crecer para dar beneficios a los asociados & 100 \\
\hline & $\begin{array}{l}\text { Tener alianzas con otras organizaciones o empresas } \\
\text { que nos compren }\end{array}$ & 100 \\
\hline \multirow{2}{*}{$\begin{array}{l}\text { Documento de } \\
\text { planeación }\end{array}$} & $\begin{array}{l}\text { Tener un documento nos ayuda a saber que } \\
\text { tenemos que hacer }\end{array}$ & 80 \\
\hline & $\begin{array}{l}\text { Consideramos que el papel realmente no cambia la } \\
\text { situación }\end{array}$ & 20 \\
\hline \multirow{3}{*}{$\begin{array}{l}\text { Necesidades } \\
\text { para ejecución } \\
\text { de la planeación }\end{array}$} & $\begin{array}{l}\text { Tener mayor conocimiento gerencial por parte de } \\
\text { todos }\end{array}$ & 98 \\
\hline & Saber cómo definirlo y ejecutarlo & 100 \\
\hline & Como comprometer y motivar a los asociados & 100 \\
\hline \multirow{2}{*}{$\begin{array}{l}\text { Lineamientos } \\
\text { para proceso de } \\
\text { planeación }\end{array}$} & Las necesidades de los asociados & 80 \\
\hline & Los estatutos & 20 \\
\hline
\end{tabular}

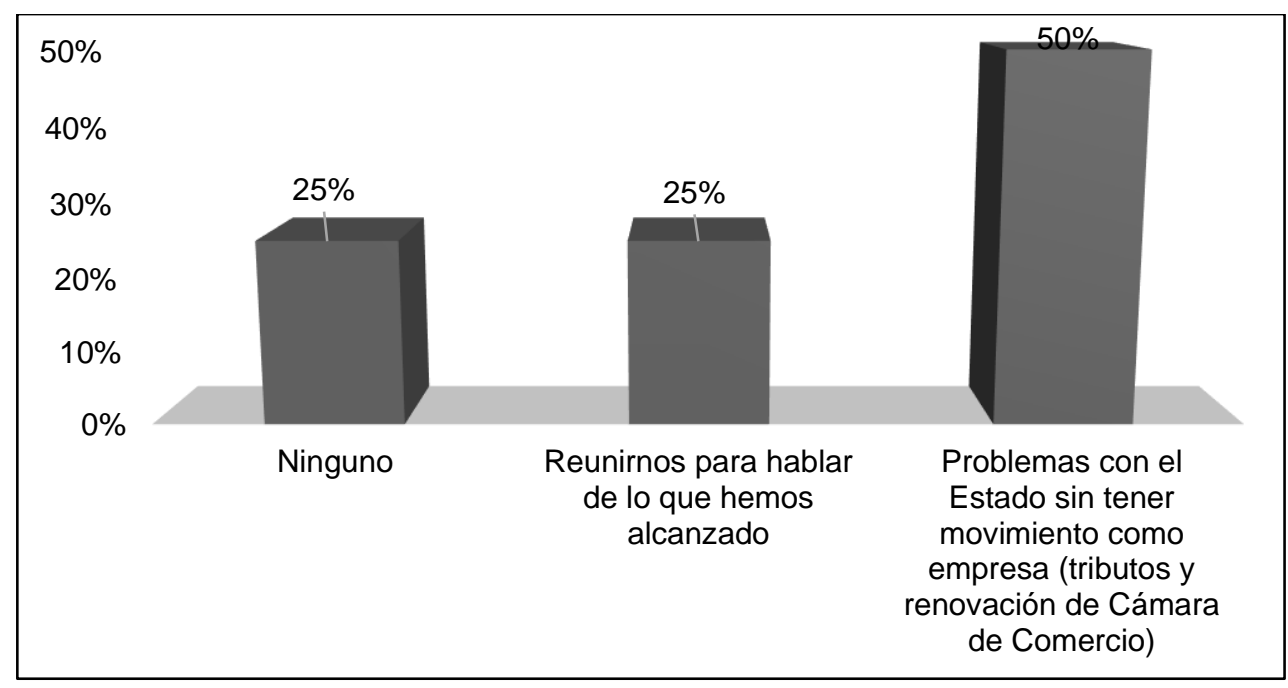

Fig. 2: Resultados de las organizaciones solidarias 


\section{DISCUSIÓN FINAL}

Se ha analizado la planificación y gestión interna de organizaciones de economía solidaria instituidas por población vulnerable usando técnicas mixtas de recolección de datos para evaluar procesos internos que influyen en la productividad y oportunidad de inclusión social de las empresas estudiadas. El documento comienza esbozando la relevancia que tiene la planificación y la gestión en el crecimiento y desarrollo futuro de las organizaciones, asimismo, la importancia de las organizaciones solidarias en el actual contexto enmarcado en la sostenibilidad y por otro lado, la situación de posconflicto que se da en el país. Por lo tanto, este modelo de organización, considerado motor de desarrollo de los territorios, debe generar capacidades y condiciones de operación interna, a fin de dar respuesta a diversos grupos de interés y al mismo tiempo ser competitivas para permanecer en el mercado y cumplir su objeto social.

Las organizaciones solidarias en población vulnerable necesitan ser capacitadas para desarrollar actividades económicas de mayores resultados en la economía local y regional; profundizar la capacitación del modelo solidario, con herramientas adecuadas al nivel de participantes y mejorar las motivaciones que impulsan a los miembros a integrar en este tipo de organizaciones, porque son personas que han pasado por eventos traumáticos, que les hacen perder el ánimo y la pasión por la vida. Por lo anterior, el apoyo requerido se estima de características diferenciadoras para lograr resultados igualmente particulares.

\section{CONCLUSIONES}

De acuerdo al trabajo presentado y a los resultados obtenidos, se pueden plantear las siguientes conclusiones principales:

1.- Las organizaciones estudiadas, surgen en el marco de un proyecto impulsado por el Estado como medio para reducir la pobreza, generando inclusión social.

2.- El proceso de planificación se ve afectado por la falta de conocimientos, competencias de directivos y miembros de las organizaciones de economía solidaria, así como carencia de recursos para capital de trabajo, deteriorando la productividad de las organizaciones.

3.- Los resultados evidencian que el carácter individualista de sus miembros, impacta en bajo desempeño y poca colaboración entre las organizaciones del sector, obstaculizando el crecimiento empresarial y proyectando un horizonte de vida incierto.

4.- Para que las organizaciones solidarias en población vulnerable puedan lograr generar ingresos y mejorar la calidad de vida de sus miembros, debe existir una coordinación interinstitucional entre lo público y lo privado, para concebir proyectos conjuntos que involucren diversos recursos y tengan mayor impacto.

\section{REFERENCIAS}

Álvarez-Aros, E.L. y C.A. Bernal-Torres, Modelo de Innovación Abierta: Énfasis en el Potencial Humano, DOI: 10.4067/S0718-07642017000100007, Información Tecnológica, 28(1), 65-76 (2017)

Alves, W., P. Ferreira y M. Araujo, Cooperativas mineras en Brasil: una visión general, Procedia Manufacturing, 13 (2017)

Arhin, A., E. Kumi y M.A.S. Adam, Facing the Bullet? Non-Governmental Organisations' (NGOs') Responses to the Changing Aid Landscape in Ghana, Voluntas, 29, 348-360 (2018)

Bretos, I. y A. Errasti, The challenges of managing across borders in worker cooperatives: Insights from the Mondragon cooperative group, Journal of Co-operative Organization and Management, 6(1), 34-42 (2018)

Cárdenas, J.C., A. Casas y N. Méndez, The Hidden Face of Justice: Fairness, Discrimination and Distribution in Transitional Justice Processes, Peace Economics, Peace Science and Public Policy, 1-28 (2014)

Cardozo, E.R., C. Rodríguez y W. Guaita, Las Pequeñas y Medianas Empresas Agroalimentarias en Venezuela y el Desarrollo Sustentable: Enfoque basado en los Principios de Manufactura Esbelta, Información Tecnológica, 22(5), 3948 (2011)

Caridad, M., P. Hernández y C. de Pelekais, Responsabilidad Gerencial: Elemento Integrador de la Sustentabilidad en la Responsabilidad Social Empresarial, Opción, 30(75) (2015)

Chareonwongsak, K., Enhancing Board Motivation for Competitive Performance of Thailand's Co-operatives, DOI: 10.1016/j.jcom.2017.01.001, Journal of Co-operative Organization and Management, 5(1), 1-13 (2017)

Cheney, G., I. Santa-Cruz, A.M. Peredo, E. Nazareno, Worker Cooperatives as an Organizational Alternative: Challenges, Achievements and Promise in Business Governance and Ownership, Organization, 21(5), 591-603 (2014) 
Chin-Shien, L. y D. Van-Thac, Untangling The Relationship Between Strategic Consistency and Organizational Performance: an Empirical Analysis of Moderator Variables, DOI:10.1017/jmo.2016.50, Journal of Management and Organization, 23(4), 483-503 (2016)

Congreso De Colombia, Ley 387 de 1997, Medidas para la Prevención del Desplazamiento Forzado, La Atención, Protección, Consolidación y Estabilización Socioeconómica de los Desplazados Internos por la Violencia en la República de Colombia, Bogotá (1997)

Dávila, J., La Doble Dimensión de una Capacidad Organizacional: Evidencias de una Organización Sin Ánimo de Lucro que Compite en el Mercado, Cuadernos de Administración, 25(44), 11-37 (2012)

Departamento Nacional De Planeación Conpes 3115, Distribución Presupuestal Sectorial para el Cumplimiento del Conpes 3057, Plan de Acción para la Prevención y Atención del Desplazamiento Forzado (2001)

Garizabal, M., M. Sánchez y H. Estrada-López, Negocios Inclusivos: Una Revisión Teórica Desde la Dinámica Colombiana, Rev. Espacios, 38(47), 6 (2017)

Garzón, M.A., Modelo de Capacidades Dinámicas, Revista Dimensión Empresarial, 13(1), 111-131 (2015)

Giordano, N. y S. Pereira, Políticas de Desenvolvimento Territorial no Meio Rural Brasileiro: Novas Institucionalidades e Protagonismo dos Atores DADOS, Revista de Ciências Sociais, 54 (2011)

Hailey, J. y M. Salway, New routes to CSO sustainability: the strategic shift to social enterprise and social investment, DOI: 10.1080/09614524.2016.1188886, Developmentlin Practice, 26, (2016)

Hambrick, D.C., S.E. Humphrey y A. Gupta, Structural Interdependence within Top Management Teams: A Key Moderator of Upper Echelons Predictions, DOI:10.1002/smj.2230, Strategic Management Journal, 36, 449-461 (2015)

Heras, I.S., The ties that bind? Exploring the Basic Principles of Worker-Owned Organizations in Practice, Organization, 21(5), 645-665 (2014)

Hernández, H.G., D.A. Cardona y J.L. Del Rio, Direccionamiento Estratégico: Proyección de la Innovación Tecnológica y Gestión Administrativa en las Pequeñas Empresas, DOI:10.4067/S0718-07642017000500003, Información Tecnológica, 28(5), 15-22 (2017)

Hernández, S.J. y R.J. Ricardo, Sector Cooperativo en Colombia, una Reflexión Crítica Desde el Sur-Oriente del Tolima, DOI: 10.17081/dege.7.2.1184, Desarrollo Gerencial, 7(2), 119-135 (2016)

Iglesias, Á., La Planificación Estratégica Como Instrumento de Gestión Pública en el Gobierno Local: Análisis de Caso, DOI: 10.5295/cdg.100156ai, Cuadernos de Gestión, 10(1), 101-119 (2010)

Lang, T. y R. Ramírez, Building New Social Capital with Scenario Planning, DOI:10.1016/j.techfore.2017.06.011, Technological Forecasting and Social Change, 124, 51-65 (2017)

Lecoutere, E., The Impact of Agricultural Co-operatives on Women's Empowerment: Evidence from Uganda, DOI: 10.1016/j.jcom.2017.03.001, Journal of Co-operative Organization and Management, 5(1), 14-27 (2017)

Mariño-Arévalo, A. y M. Valencia-Toro, Participación de la Gran Empresa en la Política Pública de Atención a las Víctimas del Conflicto Armado en Colombia, Cuadernos de Administración, 28(50), 159-185 (2015)

Papke-Shields, K.E. y K.M. Boyer-Wright, Strategic Planning Characteristics Applied to Project Management, DOI: 10.1016/j.jproman.2016.10.015, International Journal of Project Management, 35(2), 169-179 (2017)

Royer, A., J. Bijman y G. Abebe, Cooperatives, Partnerships and the Challenges of Quality Upgrading: A Case Study from Ethiopia, DOI: 10.1016/j.jcom.2017.04.001, J. Co-operative Organization and Management, 5(1), $48-55$ (2017)

Serna, H., Gerencia Estratégica: Planeación y Gestión-Teoría y Metodología, 10ª Ed., 3R Editores, Bogotá, Colombia (2008)

Sommerfeldt, E.J. y A. Yang, Relationship Networks as Strategic Issues Management: An Issue-Stage Framework of Social Movement Organization Network Strategies, DOI:10.1016/j.pubrev.2017.06.012, Public Relations Review, 43(4), 829-839 (2017)

Svensson, P.G., Organizational Hybridity: A Conceptualization of How Sport For Development and Peace Organizations Respond to Divergent Institutional Demands, DOI:10.1016/j.smr.2017.03.004, Sport Management Review (2017)

Vishnevskiy, K., O. Karasev y D. Meissner, Integrated Roadmaps for Strategic Management and Planning, DOI: 10.1016/j.techfore.2015.10.020, Technological Forecasting and Social Change, 110, 153-166 (2016)

Yildiz, Ö., J. Rommel y otros seis autores, Renewable Energy Cooperatives As Gatekeepers or Facilitators? Recent Developments in Germany and a Multidisciplinary Research Agenda, Energy Research y Social Science, 6, 59-73 (2015) 